\title{
The characteristics of the expression of heat shock proteins and cox-2 in the liver of hamsters infected with Clonorchis sinensis, and the change of endocrine hormones and cytokines
}

\author{
WonHyung Choi and JongPhil Chu
}

Department of Medical Zoology, Kyung Hee University School of Medicine, 26 Kyunghee-daero, Dongdaemun-gu, Seoul 130-701, Republic of Korea

\begin{abstract}
The liver fluke Clonorchis sinensis (Digenea) is a high-risk parasite that causes serious diseases such as cirrhosis, carcinogenic liver damage and clonorchiasis in East Asia. This study was conducted to evaluate the relationship between stress/endocrine hormones and inflammation induced by infection as well as the expression of heat shock proteins (hsp-27, hsp-90), cox-2 and cytokines in the livers of hamsters infected with $C$. sinensis. The average body weight of infected hamsters decreased up to $25 \%$ compared with that of the control group, and bile duct hyperplasia with inflammation, liver fibrosis and hepatic necrosis were observed in $C$. sinensis-infected livers. The expression of hsp-27, hsp-90, and cox-2 was significantly increased in the livers of $C$. sinensisinfected hamsters compared with the control group. Moreover, the expression levels of inflammatory cytokines (IL-1 $\beta$, IL-2, TGF- $\beta 2$ and IFN- $\alpha 1$ ) were markedly increased in the livers of the infected group compared with those of the control group. Consistently, plasma IL-3 and IL-6 levels gradually increased during the infection period, and the concentration levels of testosterone, luteinizing hormone (LH), follicle-stimulating hormone (FSH), corticosterone, and adrenocorticotropic hormone (ACTH) in C. sinensis-infected hamsters increased over $25 \%$, compared with those of the uninfected normal group. These results demonstrate that $C$. sinensis infection may increase the expression of hsp27, hsp90 and cox-2 as well as it may cause periductal fibrosis, chronic inflammation and hepatic necrosis in the liver. Furthermore, the results indicate that $C$. sinensis infection induces not only stress-induced hormone imbalance but also the sustained secretion of inflammatory cytokines through chronic stress/stimuli.
\end{abstract}

Keywords: liver fluke, Digenea, cytokines, cox-2, heat shock proteins, inflammation

Clonorchis sinensis (Cobbold, 1875), the liver fluke, is a high-risk factor of cholangiocarcinoma (CHCA) and liver cancer. It also causes serious diseases such as cholangiofibrosis, atypical adenomatous hyperplasia and cirrhosis as well as malignant neoplasm of intrahepatic bile ducts and clonorchiasis in East Asia (Kim 1984, Sher et al. 1989, Lee et al. 1994). The symptoms caused by C. sinensis range from chronic inflammation, mucosal hypertrophy, liver fibrosis, cell infiltration and abscesses to gall stone formation (Rim 2005). Clonorchis sinensis may also contribute to biliary cirrhosis and pyogenic cholangitis (Srivatanakul et al. 2004). Clonorchis sinensis is a parasite, which induces the highest rate of infectious hepatic diseases compared with other parasites in South Korea. However, the prevalence and infection rate of $C$. sinensis indicate a significant difference between endemic areas and capital city (Cho et al. 2008, Kim et al. 2009, Choi et al. 2010). Moreover, mortality of CHCA accounts for $5.8 \%$ of primary liver cancers and the proportion of CHCA among cases of liver cancer varies with areas known as endemic areas of $C$. sinensis in South Korea (Lim et al. 2006).
The inflammatory response caused by infection with another liver fluke, Opisthorchis viverrini (Poirier, 1886), was reported to play a critical role in inflammation-mediated pathogenesis and carcinogenesis (Pinlaor et al. $2004 \mathrm{a}, \mathrm{b})$. Chronic inflammation induced by $O$. viverrini infection has also been postulated to be a risk factor for CHCA and liver cancers (Coussens and Werb 2002, Ohshima et al. 2003).

Cytokines play an important role as intercellular mediators between cells of multi-cellular organisms. They also regulate the growth, differentiation, survival mechanism and various functions of cells. Furthermore, inflammatory cytokines such as IL-2, IL-3, IL- 6 and IFN- $\alpha 1$ act as mediators of the immune response (Bekisz et al. 2004, Singh et al. 2007, Lee et al. 2011, Qin et al. 2012). In particular, interleukin-1 $\beta$ (IL-1 $\beta$ ) is produced by macrophages as a mediator of the inflammatory response (Smirnova et al. 2002, Matsui et al. 2011, Samy and Igwe 2012). Interleukin-2 (IL-2) is produced by macrophages and T lymphocytes ( $\mathrm{T}$ cells) through autocrine induction during the immune response, which regulates T cell development in the thymus (Thornton et al. 2004, Peruhype-Magalhães et al. 2006, Melby et al. 2001, Seki et al. 2011). 
In addition to these cytokines, endocrine hormones act as critical modulators of homeostasis and are associated with the interactions between cytokines related to disease. Adrenocorticotropic hormone (ACTH) and corticosterone are stress hormones that are secreted by the adrenal glands and the anterior pituitary gland, respectively, in response to stress (Heiblum et al. 2000, Dikić et al. 2011). Testosterone and estradiol are sex hormones that are secreted in both testes and ovaries (Mooradian et al. 1987). Testosterone is regulated by the interaction of luteinizing hormone (LH) and follicle-stimulating hormone (FSH) produced from the pituitary gland, and estradiol is produced in the adrenal cortex as an active metabolic product of testosterone (Pentikäinen et al. 2000). FSH and LH are reproductive hormones that are produced by the anterior pituitary gland in females and males, and $\mathrm{LH}$ affects the production of testosterone (Knol 1991, Chandrashekar et al. 1994, Caraty et al. 1997).

Among proteins related to inflammation, cox-2 is not expressed in normal cells or under normal conditions, but is rapidly expressed during inflammatory periods and in neoplastic tissues.

Despite various recent studies on parasitic infections (Alves et al. 2006, Herbert et al. 2008, Sripa et al. 2009, Choi et al. 2012, Lvova et al. 2012), the correlation between chronic inflammation, stress and endocrine hormones associated with $C$. sinensis infection has not yet been reported. For this reason, the present study was conducted to evaluate the changes in cytokine and stress/endocrine hormone levels induced by $C$. sinensis infection and to identify the relationship between the expression of inflammatory cytokines and cox-2/stress proteins in the livers of $C$. sinensis-infected hamsters.

\section{MATERIALS AND METHODS}

\section{Materials}

Bovine serum albumin (BSA) and sodium dodecyl sulfate (SDS) were purchased from Sigma Chemical Co., Ltd. (St. Louis, MO). ECL western blotting detection kit was purchased from Millipore Korea Co., Ltd. All other chemicals and reagents were purchased from Merck Chemical Co., Ltd (Darmstadt, Germany) and Sigma Chemical Co., Ltd. (St. Louis, MO).

\section{Preparation of parasites}

We captured freshwater fish Pseudorasbora parva Temminck et Schlegel (Cyprinidae), which serves as second intermediate host of C. sinensis in Korea, in the Nakdong River Basin known as an endemic area of clonorchiasis in South Korea. The whole muscles of the fish were removed and digested in artificial gastric solution for $2 \mathrm{~h}$ at $37^{\circ} \mathrm{C}$. The digested material was filtered with a sieve $(0.147 \mathrm{~mm}$ diameter $)$ and washed for several times with saline. The metacercariae of $C$. sinensis were identified under a stereoscopic microscope and viable cysts were used to infect hamsters.

\section{Animals}

Syrian golden hamsters (male, 50-60 g, 4 weeks old, $\mathrm{n}=120$ ) were purchased from DaeHan Bio-Link Co., Ltd. Korea and kept in a central animal care facility under a $12 \mathrm{~h}$-light/dark cy-

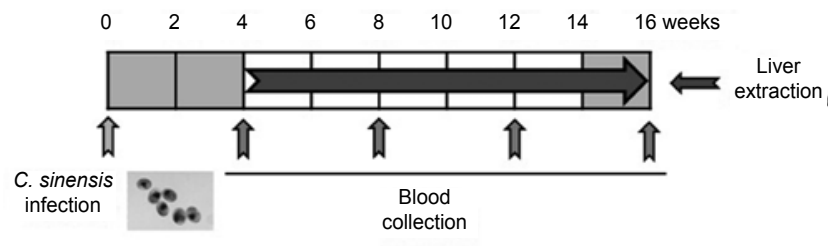

Fig. 1. Experimental design of the study in hamsters $(n=60)$ infected with metacercariae of Clonorchis sinensis. Blood samples were collected ( $n=6 /$ each group) every four weeks for 16 weeks (total $\mathrm{n}=60$, control group $\mathrm{n}=30$ and infected group $\mathrm{n}=30$ ) after $C$. sinensis infection. Livers were extracted from control $(\mathrm{n}=20)$ and infected groups $(\mathrm{n}=40)$ at 16 weeks.

cle at a controlled temperature $\left(22 \pm 0.5^{\circ} \mathrm{C}\right)$ for 16 weeks. Food and water were provided ad libitum. The facility was maintained strictly in accordance with the National Institutes of Health Guide for the Care and Use of Laboratory Animals. The animals were divided into the normal and experimental groups, and each hamster in experimental groups (infected groups) was orally infected with 50 C. sinensis metacercariae. The body weight of all hamsters was recorded before the collection of blood samples at each time point every four weeks for 16 weeks.

\section{Histopathological study}

Livers were extracted from each group at 16 weeks after C. sinensis infection (Fig. 1), fixed in $10 \%$ buffered formalin and paraffin sections were prepared using standard histological methods. All sections were cut at approximately $4.5 \mu \mathrm{m}$ and stained with haematoxylin and eosin (H\&E) for microscopic observation.

\section{Western blot analysis}

Livers were extracted from control (called herein as "normal") and infected group at 16 weeks after $C$. sinensis infection. The liver tissues were washed twice with cold PBS and lysed using RIPA lysis buffer (Elpis Biotech) and homogenization. Tissue lysate was sonicated five times at $30 \mathrm{~s}$ intervals, and it was stored at $-70^{\circ} \mathrm{C}$. Protein concentrations were measured at $595 \mathrm{~nm}$ using the Bio-Rad protein assay kit (GenDEPOT Co., Ltd., Barker, TX) and 1\% BSA as standard. Equal amounts of protein were loaded onto $12 \%$ SDS-PAGE and transferred to a nitrocellulose membrane. Membranes were blocked with $5 \%$ skim-milk in PBS containing 0.05\% Tween-20 for $1 \mathrm{~h}$ at room temperature, and they were also washed with PBS containing $0.05 \%$ Tween-20 for $5 \mathrm{~min}$ (five times). The membranes were incubated with the corresponding primary antibodies to Cox$2(1: 1000)$, Hsp $90(1: 1000)$, Hsp $27(1: 1000)$ and $\beta$-actin $(1: 10000)$ respectively, overnight at $4{ }^{\circ} \mathrm{C}$. After washing by PBS containing $0.05 \%$ Tween- 20 for $5 \mathrm{~min}$ (five times), they were incubated with goat anti-rabbit or rabbit anti-mouse IgG HRP conjugated secondary antibody for $90 \mathrm{~min}$ at room temperature. After repeating the washing step, antibody-bound protein bands were visualized using the ECL western blotting detection kit. All antibodies were purchased from Cayman Chemical (Ann Arbor, MI), Abcam (San Francisco, CA), and Cell Signaling (Cell Signaling Technology, Inc., Danvers, MA).

\section{RNA extraction and mRNA expression analysis}

The total RNA was isolated from liver tissue using an RNeasy mini extraction kit (Qiagen, Inc., South Korea) according to the manufacturer's protocol. Concentrations of 
RNA in samples were quantified using a Nano-drop (Thermo Fisher Scientific) apparatus. Reverse transcription polymerase chain reactions (RT-PCR) were conducted using a commercial kit (OneStep RT-PCR kit, Qiagen, Inc.) and a Genius thermal cycler (Bio-Rad) according to the standard protocol of the manufacturer. GenBank accession numbers for the hamster cytokine and $\beta$-actin genes used for primer design in this study were: IL-1 $\beta$ (AB028497), IL-2 (EU729351), IFN- $\alpha 1$ (S78750), TGF- $\beta 2$ (AY007214) and $\beta$-actin (DQ403055). The primer sequences $\left(5^{\prime}\right.$ to $\left.3^{\prime}\right)$ for their levels of expression are as follows: IL-1 $\beta$ (sense primer, GGTGGTGTCAGTCATTGTGG; antisense primer, AGACAGCACGAGGCATTTCT), IL-2 (sense primer, CATCCTGTCTTGCACTGACG; antisense primer, GCCTTCTTGGGCATGTAAAA), IFN- $\alpha 1$ (sense primer, ATCAACAGCAATGGGCAGGCTC; antisense primer, GCTGCTGGTAGAGGCCAGTGC), TGF- $\beta 2$ (sense primer, CAGCGCTACATCGATAGCAA; antisense primer, CCACTGCTATGTTGGGAGGT), and $\beta$-actin (sense primer, GTCGTACCACTGGCATTGTG; antisense primer, GTCACGCACAATTTCCCTCT). The level of gene expression for each sample was quantified by measuring the intensity of light emitted from correctly sized bands under ultraviolet light using a Bio-Rad Chemi-Doc. As a control for equal amounts of gene expression for each sample, the ubiquitously expressed $\beta$-actin was used.

\section{Measurement of IL-3 and IL-6}

Animals were divided into control $(\mathrm{n}=30)$ and infected groups $(n=30)$. Blood samples were collected from the left ventricle of anaesthetized hamsters into heparinized syringes at each time point ( $n=6 /$ each group) every four weeks for 16 weeks after the oral inoculation of $C$. sinensis, and the body weight of the hamsters was recorded before the collection of blood sample. Plasma was separated by centrifugation at $12000 \mathrm{rpm}$ for $15 \mathrm{~min}$ at $4{ }^{\circ} \mathrm{C}$ and stored in vials at $-70^{\circ} \mathrm{C}$ until analysis. Plasma IL-3 and IL-6 levels were measured using ELISA kits purchased from MyBioSource (San Diego, CA) and Biocompare Inc (Atlanta, GA), respectively. The activity of cytokines was measured according to the manufacturer's protocol.

Hormone assay for estradiol, testosterone, corticosterone, LH, FSH and ACTH

Animals were divided into control $(n=20)$ and infected groups $(n=40)$. Blood samples were collected as described above. Plasma was separated by centrifugation at $12000 \mathrm{rpm}$ for $15 \mathrm{~min}$ at $4{ }^{\circ} \mathrm{C}$ and stored in vials at $-70^{\circ} \mathrm{C}$ until analysis. The plasma levels of testosterone, corticosterone and estradiol were measured using radioimmunoassay kits (Siemens Medical Solution Diagnostics, CA), and the concentration levels of LH and FSH were determined using commercial radioimmunoassay kits (MP Biomedicals, Santa Ana, CA). ACTH was measured using a commercially available ELISA kit (Calbiotech, Spring Valley, CA). All hormone levels were measured according to the manufacturer's protocol.

\section{Statistical analysis}

All results are expressed as the mean \pm standard deviation (SD) of three independent experiments. Statistical analysis of the data was performed by Student's $t$-test and one-way analysis of variance (ANOVA). Values of $* P<0.05$ were considered to indicate statistically significant difference.

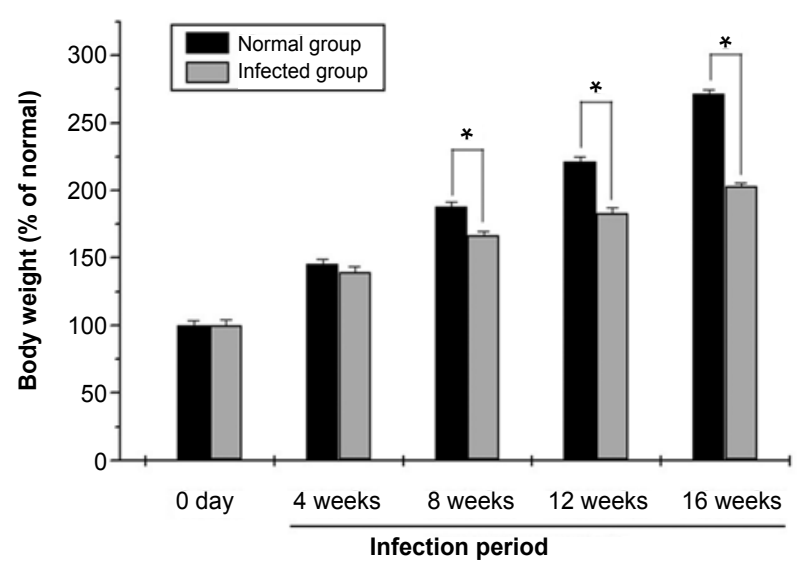

Fig. 2. Changes of the average body weight in hamsters $(n=120)$. Results are expressed as a percentage of the normal group at the beginning of the experiment, and all data are presented as the mean \pm S.D. $* P<0.05$ indicates a statistically significant difference between the normal and Clonorchis sinensisinfected group.

\section{RESULTS}

\section{Changes of body weight}

At 16 weeks post infection, the average body weight of hamsters in the group infected with Clonorchis sinensis was up to $25 \%$ lower compared with that of the control group (Fig. 2). These results indicate that $C$. sinensis infection decreases energy metabolism and muscle growth in hamsters.

\section{Histological changes in the livers}

As shown in Fig. 3A,B, the liver of hamster infected with $C$. sinensis shows morphological changes such as severe inflammation, ulceration and hepatic necrosis. Furthermore, Fig. 3C,D shows the histopathological changes including the bile duct hyperplasia with chronic inflammation, hyperplasia with inflammation and periductal fibrosis in the liver of $C$. sinensis-infected hamster. Adult worms were observed in the hepatic bile duct of infected hamsters. In contrast, the above mentioned changes were not observed in the normal group. These results demonstrate that $C$. sinensis infection may induce chronic inflammation and periductal fibrosis and also tissue damage and necrosis in the liver.

\section{Expression of heat shock proteins and cox-2}

Cox-2, hsp27 and hsp90 levels were significantly increased in the livers of $C$. sinensis-infected hamsters compared with those of the normal group (Fig. 4). These results indicate that $C$. sinensis infection increased expression levels of cox-2 associated with chronic inflammation involving periductal fibrosis. The infection also induced the activation of hsp27 and hsp90 in the liver as 

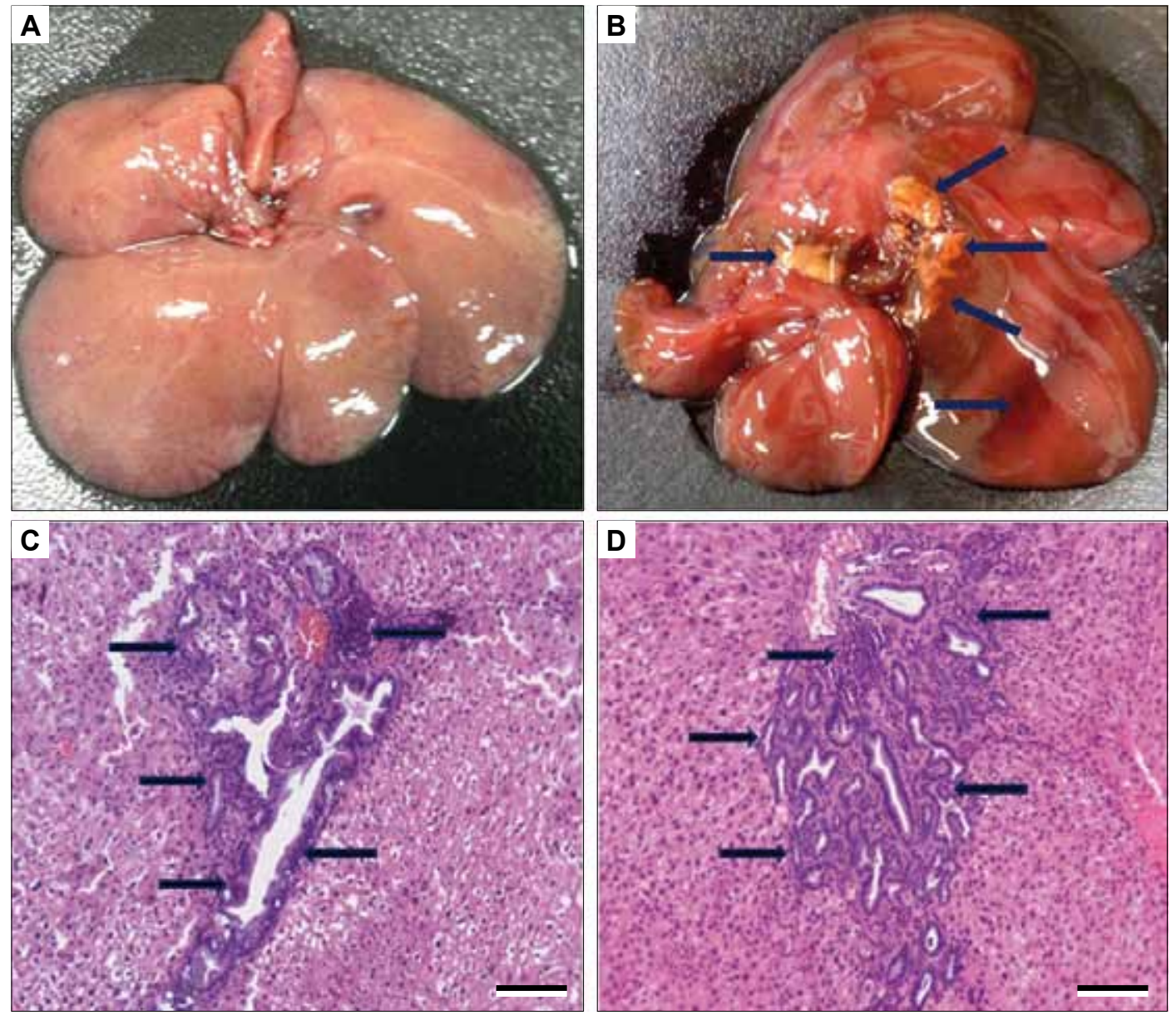

Fig. 3. Morphological and histopathological changes of the liver in the normal and Clonorchis sinensis-infected groups. A - liver tissue of a normal hamster; B - liver tissue of a C. sinensis-infected hamster (arrows indicate the inflamed region and necrotic region), C, D - bile duct hyperplasia with chronic inflammation and periductal fibrosis in the livers of $C$. sinensis-infected hamsters (arrows) $(\mathrm{H} \& \mathrm{E}$, scale $\mathrm{bar}=250 \mu \mathrm{m})$. The livers were extracted from the normal and infected groups at 16 weeks after $C$. sinensis infection.

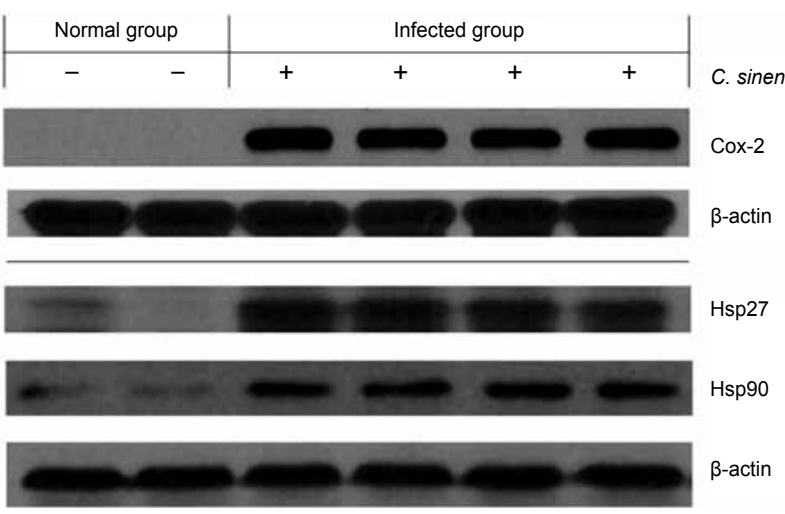

Fig. 4. The change of the expression levels of Hsp-27, Hsp-90 and Cox-2 in the liver of the normal hamsters and those infected with Clonorchis sinensis. Proteins were isolated from liver tissue of the normal and infection groups at 16 weeks after $C$. sinensis infection, and protein expression was measured using western blot analysis.

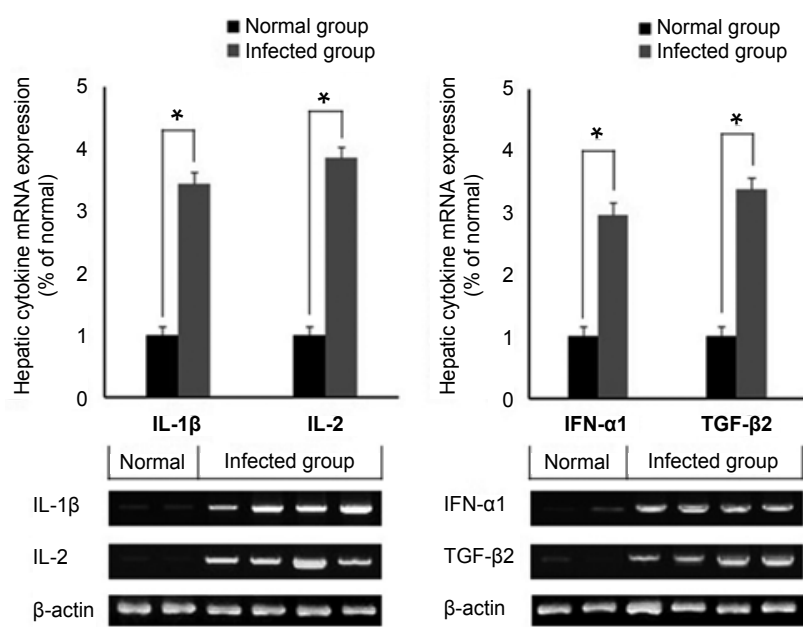

Fig. 5. RT-PCR analysis of the expression levels of cytokine genes in the livers of the normal and Clonorchis sinensis-infected groups. The total RNA was isolated from liver tissue of the normal $(\mathrm{n}=20)$ and infection groups $(\mathrm{n}=40)$ at 16 weeks after C. sinensis infection. Results are expressed as a percentage of the normal group, and all data are presented as the mean \pm S.D. ${ }^{*} P<0.05$ indicates a statistically significant difference between the normal and infection group. 
a response to the stress stimuli and tissue damage during the period of the experiment.

\section{Expression of inflammatory cytokines}

As shown in Fig. 5, the expression levels of IL-1 $\beta$, IL-2, TGF- $\beta 2$ and IFN- $\alpha 1$ markedly increased in $C$. sinensisinfected hamsters compared with those of the normal group. These results demonstrate that $C$. sinensis infection induces the secretion and production of IL-1 $\beta$, IL-2, TGF- $\beta 2$ and IFN- $\alpha 1$, which are rapidly activated by infections, inflammation and tissue damage in the liver.

\section{Expression levels of IL-3 and IL-6 cytokines}

As shown in Fig. 6, the plasma levels of IL-3 and IL-6 were markedly increased in the infected group compared with those of the normal group in a time-dependent manner after $C$. sinensis infection. In particular, the levels of IL-6 increased compared with those of IL-3 at 12 weeks.
These results show that $C$. sinensis infection markedly increases the secretion of IL- 3 and IL- 6 as well as the activation of the immune network induced by the infiltration of lymphocytes, inflammation and tissue damage.

\section{Changes in endocrine hormone levels}

The plasma levels of LH, FSH, testosterone, corticosterone and ACTH in C. sinensis-infected group were higher than those of the normal group, whereas levels of estradiol were similar or lower than that of the normal group (Fig. 7). Furthermore, the levels of LH, FSH, testosterone, corticosterone and $\mathrm{ACTH}$ in the infection group increased over $36 \%, 31 \%, 27 \%, 70 \%$ and $28 \%$, respectively, compared with those of the normal group. These results demonstrate that chronic stress/stimuli such as chronic inflammation and liver lesions or necrosis caused by $C$. sinensis infection act as factors that induce the imbalance of endocrine hormones by stimulating the pituitary gland (Fig. 8).
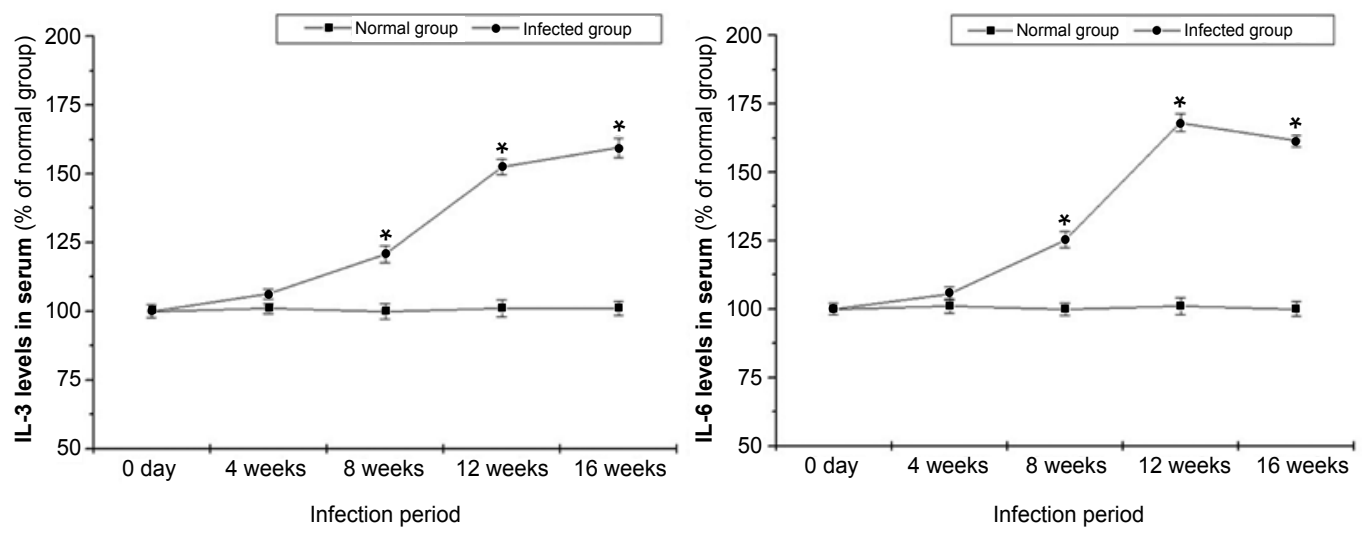

Fig. 6. Plasma levels of inflammatory cytokines (IL-3 and IL-6) in the normal and infection groups. Blood samples were collected from the animals (total $n=60, n=6 /$ normal group, $n=6 /$ infection group) every four weeks for 16 weeks after Clonorchis sinensis infection. Data are presented as mean values of the animals $(\mathrm{n}=6)$ per each group at each time point, and the results are expressed as a percentage of the normal group. ${ }^{*} P<0.05$ indicates a statistically significant difference between the normal and $C$. sinensisinfected group.
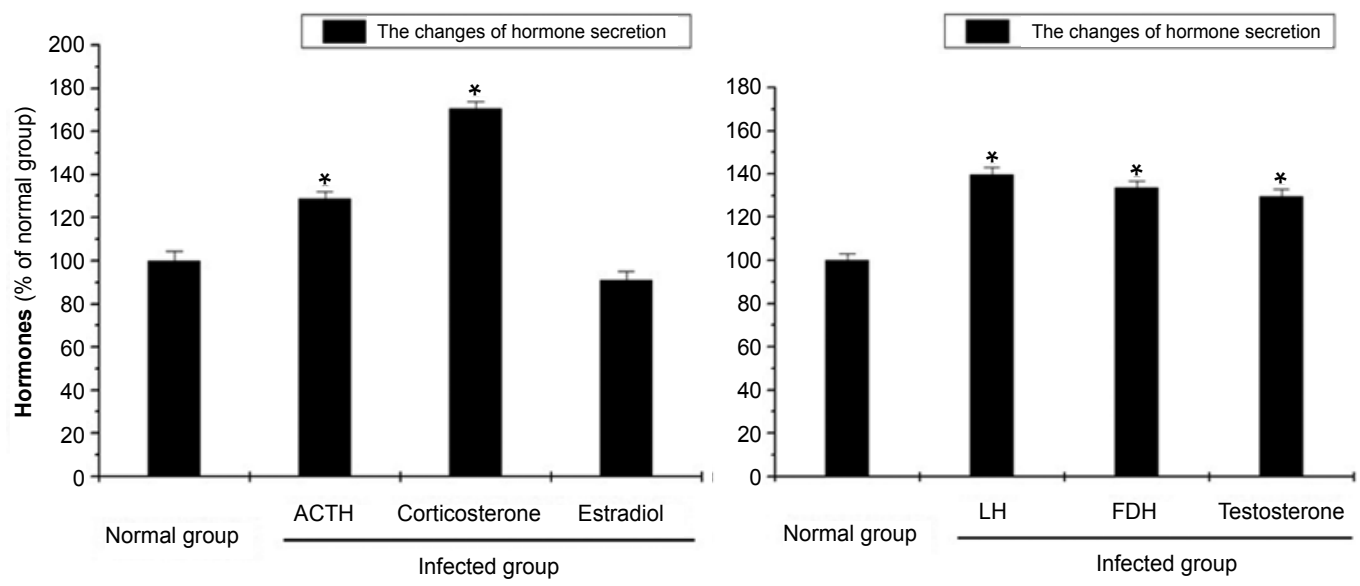

Fig. 7. The concentration levels of endocrine hormones in the normal and infection groups. Blood samples were collected from the animals ( $n=20$ /normal group, $n=40 /$ infection group) at 16 weeks after Clonorchis sinensis infection. Results are expressed as a percentage of the normal group, and all data are presented as the mean \pm S.D. $* P<0.05$ indicates a statistically significant difference between the normal and $C$. sinensis-infected group. 


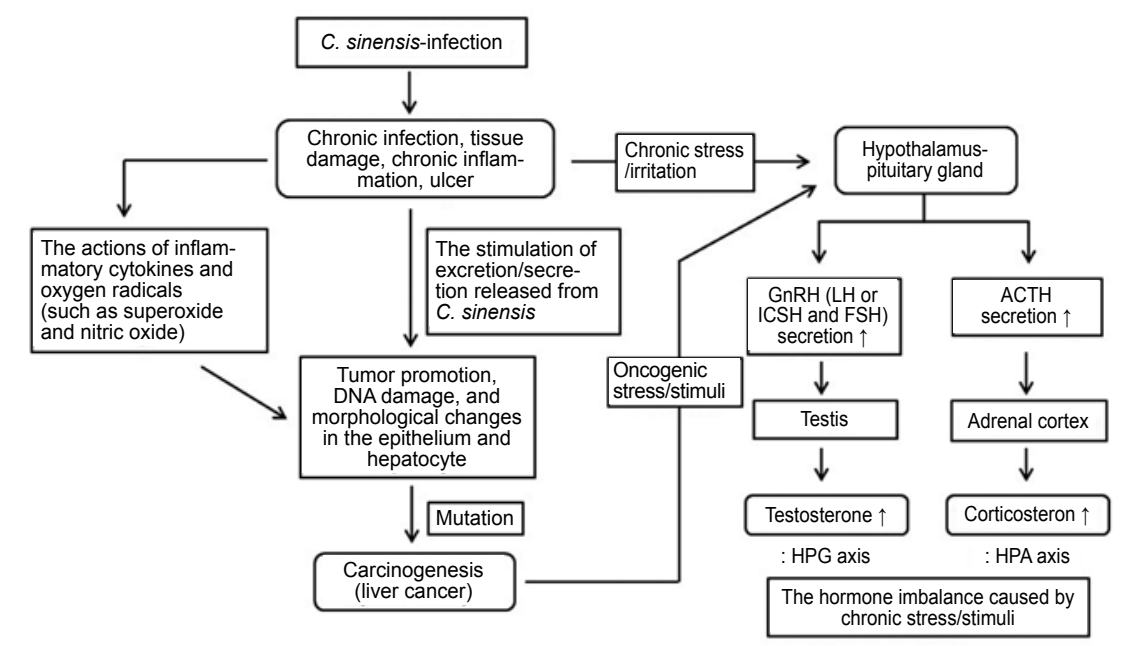

Fig. 8. A hypothetical overall signaling mechanism for the change in endocrine hormones and the oncogenic transformation in the liver in the group infected with Clonorchis sinensis. Hypothalamic-pituitary-gonadal axis (HPG axis), hypothalamic-pituitaryadrenal axis (HPA axis), gonadotropin releasing hormone (GnRH), and interstitial cell stimulating hormone (ICSH).

\section{DISCUSSION}

From a public health perspective, the ingestion of raw fish can cause parasitic infections such as fish-borne trematodes and anisakiasis. Clonorchis sinensis infection is caused by the ingestion of raw fish containing metacercariae of this liver fluke. Small liver flukes, i.e. C. sinensis and Opisthorchis spp., widely cause helminthic diseases such as multiple nodules in hepatocellular carcinoma, chronic clonorchiasis, pyogenic cholangitis, periductal fibrosis and biliary cirrhosis. According to WHO, 17.5 million people worldwide were infected with liver flukes (9 million with $O$. viverrini, 7 million with $C$. sinensis, and 1.5 million with O.felineus) in 1995 (IARC Working Group 1994, Moller et al. 1995), and 680.8 million people were reported to be exposed to the risk of liver fluke infection (C. sinensis, O. viverrini and O. felineus) in 2005 (Keiser and Utzinger 2005). Other authors reported that 35 million people were infected with $C$. sinensis globally, of whom 15 million people occurred in China (Lun et al. 2005). As mentioned above, the prevalence of liver fluke infection, which represents a major risk factor for cholangiocarcinoma, seems to be increasing (Sher et al. 1989, Lee et al. 1994, Watanapa and Watanapa 2002).

Opisthorchis viverrini is known to induce persistent infection that causes complications, including the infiltration of lymphocytes, chronic inflammation, fibrosis, adenomatous hyperplasia of bile duct epithelium and neoplasm of the bile duct (Prakobwong et al. 2009). Fibrosis is the result of chronic inflammatory reactions that are induced by a variety of stimuli, including persistent infection (Wynn 2008).

Reactive nitrogen and oxygen are generated during infection in the livers of hamsters infected with $O$. viverrini, and the resulting DNA damage and mutation contribute to carcinogenesis that is associated with chronic inflamma- tion in the liver (Pinlaor 2003). Furthermore, CHCA that is concomitant with clonorchiasis is relatively common in endemic areas of $C$. sinensis. Bouvard et al. (2009) reported the influence and level of dangerousness concerning parasitic infection as carcinogenic factors that cause cancer development. According to that report, C. sinensis was classified into Risk Group 1 as a carcinogenic parasite factor that causes liver cancer in humans (Bouvard et al. 2009) and $C$. sinensis infection was reported as a higher risk factor than hepatitis $\mathrm{B}$ and $\mathrm{C}$ viruses that induce cholangiocarcinoma (Shin et al. 2010). The correlation between cancer and parasitic infection is gradually being confirmed by new diagnostic techniques for pathogenic infections and understanding concerning molecular mechanisms of cancer development.

In the present study, the histopathological changes such as bile duct hyperplasia with chronic inflammation and liver fibrosis as well as hepatic necrosis and severe inflammation were observed in the liver of the infected group. Cox-2 is expressed in the cells where prostaglandins are up-regulated during the inflammatory response, which is activated in various cancer cells and inflamed tissues (Williams et al. 1999, Fournier and Gordon 2000, Gately 2000, Gupta and Dubois 2001, Simmons et al. 2004). Overexpression of cox-2 increases the proliferation of hepatocellular carcinoma cells, dysplastic nodule growth and invasiveness, but also causes the interaction with several proteins such as p-Akt, VEGF and iNOS, which play a key role in carcinogenesis (Koga et al. 1999, Kondo et al. 1999, Rahman et al. 2001, Leng et al. 2003, Tang et al. 2005).

The expression of HSPs is induced by various stressors, including infection, inflammation, hypoxia and dehydration (Santoro 2000). HSPs not only function as intracellular chaperones for other proteins, but also as key pro- 
teins associated with cardiovascular system (Benjamin and McMillan 1998, Borges and Ramos 2005). Tissue damage induced by parasites accelerates the activation of immunocytes (Doetze et al. 2000, Herbert et al. 2008, Nieuwenhuizen et al. 2009). As mentioned above, chronic stress stimuli, lesions and tissue damage of the liver induced by $C$. sinensis infection increased the expression of cox-2, hsp27 and hsp90 in the liver.

Inflammatory cytokines play pivotal roles in various immune responses that are activated by pathogen infection, inflammation and tissue damage. IFN- $\gamma$ and IL-2 are markedly increased in BALB/c mice and rats infected with C. sinensis (Quan et al. 2002, Choi et al. 2003). IFN- $\alpha$ regulates the fine tuning of cellular responses for pathogen defence (Moll et al. 2011). Furthermore, TGF- $\beta$ in normal cells stops the cell cycle at the G1 phase and induces the death of impaired cells. However, the expression of TGF- $\beta$ in cancer cells is markedly increased compared with that of normal cells, and it not only causes immunosuppression but also promotes rapid proliferation of cancer cells (Hanahan and Weinberg 2000, Konrad et al. 2009, Berezowski et al. 2012). TGF- $\beta$ and IL-10 mediate cellular hyporesponsiveness in patients infected with $\mathrm{On}$ chocerca volvulus and also suppress hepatic inflammation in mice infected with Schistosoma mansoni (Doetze et al. 2000, Herbert et al. 2008). In this aspect, the increased expression of inflammatory cytokines in the livers of C. sinensis-infected hamsters may be associated with liver cancer and cholangiocarcinoma induced by $C$. sinensis as well as excretory/secretory products that are produced by the parasite (Sripa et al. 2007, Pak et al. 2009).

IL-3 acts as a pleiotropic cytokine that stimulates the differentiation of multipotent haematopoietic cells and NK cells, and IL-6 is an inflammatory cytokine that is secreted as a critical modulator in the host defence against pathogens. The increased levels of IL-6 in humans infected with $O$. viverrini rapidly enhance the development of periductal fibrosis in the liver (Sripa et al. 2009). However, the expression levels of IL-3 and IL-6 in C. sinensis-infected hamsters have not yet been reported. In the present study, plasma IL-3 and IL-6 levels of $C$. sinensis-infected hamsters markedly increased during the infection period with a time-dependent aspect compared with those of the normal group.

The increase in these cytokines may be a continuous response of the immune network that occurs because the immune cells gradually recognize the presence of chronic inflammation and hepatic necrosis induced by $C$. sinensis infection. In addition, the levels of endocrine hormones were markedly increased in the $C$. sinensis-infected group compared with those of the normal group. The change in the plasma level of these hormones may be the result of the persistent secretion of endocrine hormones as the hypothalamus and pituitary gland recognize chronic stress/ stimuli, including chronic inflammation and liver damage.

In summary, the results of this study demonstrate that C. sinensis infection increases the secretion of endocrine hormones such as testosterone, corticosterone, $\mathrm{FSH}, \mathrm{LH}$ and $\mathrm{ACTH}$, the expression levels of cox-2 associated with inflammatory response and the activation of hsp27 and hsp90 in the liver in response to chronic stress stimuli. The results also show that liver damage and inflammation caused by $C$. sinensis infection activate the expression of inflammatory cytokines such as IL-1 $\beta$, IL-2, TGF- $\beta 2$ and IFN- $\alpha 1$ and also consistently increase the levels of IL-3 and IL- 6 secreted by the immune network. Thus, the process of hepatic damage and cancer development induced by $C$. sinensis infection may be defined as follows: 1) induction of chronic stimuli by toxic products produced by the parasite, 2) stimulation by carcinogenic secretory proteins released from the parasite, 3) hormonal imbalance and disturbances induced by parasitic infection, 4) infiltration and hyperplasia of lymphocytes, 5) sustained production and secretion of inflammatory cytokines, 6) chronic inflammation and liver necrosis induced by infection and 7) continuation of chronic stress stimulation. The results of this study also demonstrate the correlation between the inflammation, hormone imbalance and infectious stress/stimuli, which were not reported in the previous studies on C. sinensis.

Acknowledgments. The authors would like to express sincere gratitude to the staff of the Kyung Hee University School of Medicine for providing laboratory facility and research equipment during the course of the experiment. The authors approved the final manuscript and report no conflicts of interest.

\section{REFERENCES}

Alves Oliveira L.F., Moreno E.C., Gazzinelli G., MartinsFilho O.A., Silveira A.M., Gazzinelli A., Malaquias L.C., LoVerde P., Leite P.M., Correa-Oliveira R. 2006: Cytokine production associated with periportal fibrosis during chronic schistosomiasis mansoni in humans. Infect. Immun. 74: 1215-1221.

Berisz J., Schmeisser H., Hernandez J., Goldman N.D., Zoon K.C. 2004: Human interferons alpha, beta and omega. Growth Factors 22: 243-251.
Benjamin I.J., McMillan D.R. 1998: Stress (heat shock) proteins: molecular chaperones in cardiovascular biology and disease. Circ. Res. 83: 117-132.

Berezowski P., Strzalka-Mrozik B., Forminska-Kapuscik M., Mazurek U., Filipek E., Nawrocka L., Pieczara E., BanaSIAK P., KIMSA M. 2012: Posttraumatic temporal TGF- $\beta$ mRNA expression in lens epithelial cells of paediatric patients. Folia. Biol. 58: 24-29.

Borges J.C., Ramos C.H. 2005: Protein folding assisted by chaperones. Protein. Pept. Lett. 12: 257-261. 
Bouvard V., Baan R., Straif K., Grosse Y., Secretan B., El Ghissassi F., Benbrahim-Tallaa L., Guha N, Freeman C., Galichet L., Cogliano V. 2009: WHO International Agency for Research on Cancer Monograph Working Group. A review of human carcinogens-Part B: biological agents. Lancet Oncol. 10: 321-322.

Caraty A., Miller D.W., Delaleu B., Martin G.B. 1997: Stimulation of LH secretion in sheep by central administration of corticotrophin-releasing hormone. J. Reprod. Fertil. 111: 249-257.

Chandrashekar V., Majumdar S.S., Bartke A. 1994: Assessment of the role of follicle-stimulating hormone and prolactin in the control of testicular endocrine function in adult djungarian hamsters (Phodopus sungorus) exposed to either short or long photoperiod. Biol. Reprod. 50: 82-87.

Cho S.H., Lee K.Y., Lee B.C., Cho P.Y., Cheun H.I., Hong S.T., Sohn W.M., KIM T.S. 2008: Prevalence of clonorchiasis in southern endemic areas of Korea in 2006. Korean J. Parasitol. 46: 133-137.

Choi W.H., Chu J.P., Jiang M., Lee Y.S., Kim B.S., Kim D.G., PARK Y.K. 2010: Analysis of parasitic diseases diagnosed by tissue biopsy specimens at KyungHee Medical Center (19842005) in Seoul, Korea. Kor. J. Parasitol. 48: 85-88.

Chor W.H., Jiang M.H., Chu J.P. 2012: Antiparasitic effects of Zingiber officinale (ginger) extract against Toxoplasma gondii. J. Appl. Biomed. In press. DOI: 10.2478/v10136-012-0014-y.

Choi Y.K., Yoon B.I., Won Y.S., Lee C.H., Hyun B.H., Kim H.C., Oн G.T., Kiм D.Y. 2003: Cytokine responses in mice infected with Clonorchis sinensis. Parasitol. Res. 91: 87-93.

Coussens L.M., Werb Z. 2002: Inflammation and cancer. Nature 420: 860-867.

Dikić D., Budeč M., Vranješ-Durić S., Koko V., Vignjević S., Mitrović O. 2011: The acute effect of ethanol on adrenal cortex in female rats - possible role of nitric oxide. Alcohol Alcohol 46: 523-528.

Doetze A., Satoguina J., Burchard G., Rau T., Löliger C., Fleischer B., Hoerauf A. 2000: Antigen-specific cellular hyporesponsiveness in a chronic human helminth infection is mediated by T(h)3/T(r)1-type cytokines IL-10 and transforming growth factorbeta but not by a $\mathrm{T}(\mathrm{h}) 1$ to $\mathrm{T}(\mathrm{h}) 2$ shift. Int. Immunol. 12: 623-630.

Fournier D.B., Gordon G.B. 2000: COX-2 and colon cancer: potential targets for chemoprevention. J. Cell. Biochem. Suppl. 34: 97-102.

Gately S. 2000: The contributions of cyclooxygenase-2 to tumor angiogenesis. Cancer Metastasis Rev. 19: 19-27.

Gupta R.A., Dubois R.N. 2001: Colorectal cancer prevention and treatment by inhibition of cyclooxygenase-2. Nat. Rev. Cancer. 1: $11-21$.

Hanahan D., Weinberg R.A. 2000: The hallmarks of cancer. Cell 100: 57-70.

Heiblum R., Arnon E., Gvaryahu G., Robinzon B., Snapir N. 2000: Short-term stress increases testosterone secretion from testes in male domestic fowl. Gen. Comp. Endocrinol. 120: 55-66.

Herbert D.R., Orekov T., Perkins C., Finkelman F.D. 2008: IL-10 and TGF-beta redundantly protect against severe liver injury and mortality during acute schistosomiasis. J. Immunol. 181: 7214-7220.

IARC WORKING GROUP 1994: Infection with liver flukes (Opisthorchis viverrini, Opisthorchis felineus, and Clonorchis sinensis). IARC Monogr. Eval. Carcinog. Risks. Hum. 61: 121-175.

Keiser J., Utzinger J. 2005: Emerging foodborne trematodiasis. Emerg. Infect. Dis. 11: 1507-1514.
Kim Y.I. 1984: Liver carcinoma and liver fluke infection. Arzneimittelforschung 34: 1121-1126.

Kim T.S., Cho S.H., Huh S., Kong Y., Sohn W.M., Hwang S.S., Chai J.Y., Lee S.H., Park Y.K., Oh D.K., Lee J.K. 2009: A nationwide survey on the prevalence of intestinal parasitic infections in the Republic of Korea, 2004. Korean J. Parasitol. 47: 37-47.

KNOL B.W. 1991: Stress and the endocrine hypothalamus-pituitary-testis system: a review. Vet. Q. 13: 104-114.

Koga H., Sakisaka S., Ohishi M., Kawaguchi T., Taniguchi E., Sasatomi K., Harada M., Kusaba T., Tanaka M., Kimura R., Nakashima Y., Nakashima O., Kojiro M., Kurohiji T., Sata M. 1999: Expression of cyclooxygenase-2 in human hepatocellular carcinoma: relevance to tumor dedifferentiation. Hepatology 29: 688-696.

Kondo M., Yamamoto H., Nagano H., OKami J., Ito Y., Shimizu J., Eguchi H., Miyamoto A., Dono K., Umeshita K., Matsuura N., Wakasa K., Nakamori S., Sakon M., Monden M. 1999: Increased expression of COX-2 in nontumor liver tissue is associated with shorter disease-free survival in patients with hepatocellular arcinoma. Clin. Cancer Res. 5: 4005-4012.

Konrad L., Scheiber J.A., Schwarz L., Schrader A.J., HofMANN R. 2009: TGF-betal and TGF-beta2 strongly enhance the secretion of plasminogen activator inhibitor-1 and matrix metalloproteinase- 9 of the human prostate cancer cell line PC3. Regul. Pept. 155: 28-32.

Lee J.H., Noh J., Noh G., Choi W.S., Cho S., Lee S.S. 2011: Allergen-specific transforming growth factor- $\beta$-producing $\mathrm{CD} 19(+) \mathrm{CD} 5(+)$ regulatory $\mathrm{B}-$ cell $(\mathrm{Br} 3)$ responses in hHuman late eczematous allergic reactions to cow's milk. J. Interferon Cytokine Res. 31: 441-449.

Lee J.H., Yang H.M., BaK U.B., Rim H.J. 1994: Promoting role of Clonorchis sinensis infection on induction of cholangiocarcinoma during two-step carcinogenesis. Kor. J. Parasitol. 32: $13-18$.

Leng J., Han C., Demetris A.J., Michalopoulos G.K., Wu T. 2003: Cyclooxygenase-2 promotes hepatocellular carcinoma cell growth through Akt activation: evidence for Akt inhibition in celecox-ibinduced apoptosis. Hepatology 38: 756-768.

Lim M.K., Ju Y.H., Franceschi S., Oh J.K., Kong H.J., Hwang S.S., Park S.K., Cho S.I., Sohn W.M., Kim D.I., Yoo K.Y., Hong S.T., Shin H.R. 2006: Clonorchis sinensis infection and increasing risk of cholangiocarcinoma in the Republic of Korea. Am. J. Trop. Med. Hyg. 75: 93-96.

Lun Z.R., Gasser R.B., Lai D.H., Li A.X., Zhu X.Q., Yu X.B., FANG Y.Y. 2005: Clonorchiasis: a key foodborne zoonosis in China. Lancet Infect. Dis. 5: 31-41.

Lvova M.N., Tangkawattana S., Balthaisog S., Katokhin A.V., Mordvinov V.A., Sripa B. 2012: Comparative histopathology of Opisthorchis felineus and Opisthorchis viverrini in a hamster model: an implication of high pathogenicity of the European liver fluke. Parasitol. Int. 61: 167-172.

Matsui M., Rouleau V., Bruyère-Ostells L., Goarant C. 2011: Gene expression profiles of immune mediators and histopathological findings in animal models of leptospirosis: comparison between susceptible hamsters and resistant mice. Infect. Immun. 79: 4480-4492.

Melby P.C., Chandrasekar B., Zhao W., Coe J.E. 2001: The hamster as a model of human visceral leishmaniasis: progressive disease and impaired generation of nitric oxide in the face of a prominent Th1-like cytokine response. J. Immunol. 166: 1912-1920.

Moll H.P., Maier T., Zommer A., Lavoie T., Brostjan C. 2011: The differential activity of interferon- $\alpha$ subtypes is consistent among distinct target genes and cell types. Cytokine 53: 52-59. 
Moller H., Heseltine E., Vainio H. 1995: Working group report on schistosomes, liver flukes and Helicobacter pylori. Int. J. Cancer. 60: 587-589.

Mooradian A.D., Morley J.E., Korenman S.G. 1987: Biological actions of androgens. Endocr. Rev. 8: 1-28.

Nieumenhuizen N., Herbert D.R., Brombacher F., Lopata A.L. 2009: Differential requirements for interleukin (IL)-4 and IL-13 in protein contact dermatitis induced by Anisakis. Allergy 64: 1309-1318.

Ohshima H., Tatemichi M., Sawa T. 2003: Chemical basis of inflammation-induced carcinogenesis. Arch. Biochem. Biophys. 417: 3-11.

PaK J.H., Kim D.W., Moon J.H., Nam J.H., Kıм J.H., Ju J.W., Kim T.S., Seo S.B. 2009: Differential gene expression profiling in human cholangiocarcinoma cells treated with Clonorchis sinensis excretory-secretory products. Parasitol. Res. 104: 1035-1046.

Pentikäinen V., Erkillä K., Suomalainen L., Parvinen M., Dunkel L. 2000: Estradiol acts as a germ cell survival factor in the human testis in vitro. J. Clin. Endocrinol. Metab. 85: 2057-2067.

Peruhype-Magalhães V., Martins-Filho O.A., Prata A., Silva lde A., Rabello A., Teixeira-Carvalho A., Figueiredo R.M., Guimarães-Carvalho S.F., Ferrari T.C., Van Weyenbergh J., Correa-Oliveira R. 2006: Mixed inflammatory/regulatory cytokine profile marked by simultaneous raise of interferon-gamma and interleukin-10 and low frequency of tumour necrosis factor-alpha $(+)$ monocytes are hallmarks of active human visceral Leishmaniasis due to Leishmania chagasi infection. Clin. Exp. Immunol. 146: 124-132.

PINLAOR S. 2003: 8-nitroguanine formation in the liver of hamsters infected with Opisthorchis viverrini. Biochem. Biophys. Res. Commun. 309: 567-571.

Pinlaor S., Hiraku Y., Ma N., Yongvanit P., Semba R., Oikawa S., Murata M., Sripa B., Sithithaworn P., Kawanishi S. 2004a: Mechanism of NO-mediated oxidative and nitrative DNA damage in hamsters infected with Opisthorchis viverrini: a model of inflammation-mediated carcinogenesis. Nitric Oxide 11: $175-183$.

Pinlaor S., Ma N., Hiraku Y., Yongvanit P., Semba R., Oikawa S., Murata M., Sripa B., Sithithaworn P., Kawanishi S. 2004b: Repeated infection with Opisthorchis viverrini induces accumulation of 8-nitroguanine and 8-oxo-7, 8-dihydro-20deoxyguanine in the bile duct of hamsters via inducible nitric oxide synthase. Carcinogenesis 25: 1535-1542.

Prakobwong S., Pinlaor S., Yongvanit P., Sithithaworn P., Pairojkul C., Hiraku Y. 2009: Time profiles of the expression of metalloproteinases, tissue inhibitors of metalloproteases, cytokines and collagens in hamsters infected with Opisthorchis viverrini with special reference to peribiliary fibrosis and liver injury. Int. J. Parasitol. 39: 825-835.

Qin Z.F., Hou D.Y., Fang Y.Q., Xiao H.J., Wang J., Li K.C. 2012: Interferon-alpha enhances excitatory transmission in substantia gelatinosa neurons of rat spinal cord. Neuroimmunomodulation 19: 235-240.

Quan F.S., Cно S.W., Joo K.H. 2002: Proliferation and cytokine production of lymphocytes from Clonorchis sinensis-infected rats in response to stimulators in vitro. Chin. J. Parasitol. Parasit. Dis. 20: 136-140.

Rahman M.A., Dhar D.K., Yamaguchi E., Maruyama S., Sato T., Hayashi H., Ono T., Yamanoi A., Kohno H., Nagasue N. 2001: Coexpression of inducible nitric oxide synthase and COX-2 in hepatocellular carcinoma and surrounding liver: pos- sible involvement of COX-2 in the angiogenesis of hepatitis $\mathrm{C}$ virus-positive cases. Clin. Cancer Res. 7: 1325-1332.

Rıм H.J. 2005: Clonorchiasis: an update. J. Helminthol. 79: 269281.

Samy A.S., Igwe O.J. 2012: Regulation of IL-1 $\beta$-induced cyclooxygenase- 2 expression by interactions of $A \beta$ peptide, apolipoprotein $\mathrm{E}$ and nitric oxide in human neuroglioma. J. Mol. Neurosci. 47: 533-545.

Santoro M.G. 2000: Heat shock factors and the control of the stress response. Biochem. Pharmacol. 59: 55-63.

Seki S., Nakashima H., Nakashima M., Kinoshita M. 2011: Antitumor immunity produced by the liver Kupffer cells, NK cells, NKT cells, and CD8 CD122 T cells. Clin. Dev. Immunol. 2011: 868345.

Sher L., Iwatsuki S., Lebeau G., Zajko A.B. 1989: Hilar cholangiocarcinoma associated with clonorchiasis. Dig. Dis. Sci. 34: $1121-1123$.

Shin H.R., Oh J.K., Masuyer E, Curado M.P., Bouvard V., Fang Y.Y., Wiangnon S., SRipa B., Hong S.T. 2010: Epidemiology of cholangiocarcinoma: an update focusing on risk factors. Cancer Sci. 101: 579-585.

Simmons D.L., Botting R.M., Hla T. 2004: Cyclooxygenase isozymes: the biology of prostaglandin synthesis and inhibition. Pharmacol. Rev. 56: 387-437.

Singh B., Murphy R.F., Ding X.Z., Roginsky A.B., Bell R.H. Jr, Adrian T.E. 2007: On the role of transforming growth factor-beta in the growth inhibitory effects of retinoic acid in human pancreatic cancer cells. Mol. Cancer. 6: 82 .

Smirnova M.G., Kiselev S.L., Gnuchev N.V., Birchall J.P., Pearson J.P. 2002: Role of the pro-inflammatory cytokines tumor necrosis factor-alpha, interleukin-1 beta, interleukin-6 and interleukin- 8 in the pathogenesis of the otitis media with effusion. Eur. Cytokine Netw. 13: 161-172.

Sripa B., Kaewkes S., Sithithaworn P., Mairiang E., Laha T., Smout M., Pairojkul C., Bhudhisawasdi V., Tesana S., Thinkamrop B., Bethony J.M., Loukas A., Brindley P.J. 2007: Liver fluke induces cholangiocarcinoma. PLoS Med. 4: 1148-1155.

Sripa B., Mairiang E., Thinkhamrop B., Laha T., Kaewkes S., Sithithaworn P., Tessana S., Loukas A., Brindley P.J., BeTHONY J.M. 2009: Advanced periductal fibrosis from infection with the carcinogenic human liver fluke Opisthorchis viverrini correlates with elevated levels of interleukin-6. Hepatology 50: 1273-1281.

Srivatanakul P., Sriplung H., Deerasamee S. 2004: Epidemiology of liver cancer: an overview. Asian Pac. J. Cancer Prev. 5: $118-125$.

Tang T.C., Poon R.T., Lau C.P., Xie D., Fan S.T. 2005: Tumor cyclooxygenase-2 levels correlate with tumor invasiveness in human hepatocellular carcinoma. World J. Gastroenterol. 11: 1896-1902.

Thornton A.M., Donovan E.E., Piccirillo C.A., Shevach E.M. 2004: Cutting edge: IL-2 is critically required for the in vitro activation of $\mathrm{CD} 4+\mathrm{CD} 25+\mathrm{T}$ cell suppressor function. J. Immunol. 172: 6519-6523.

WATANAPA P, Watanapa W.B. 2002: Liver fluke-associated cholangiocarcinoma. Brit. J. Surg. 89: 962-970.

Williams C.S., Mann M., DuBois R.N. 1999: The role of cyclooxygenases in inflammation, cancer, and development. Oncogene 18: 7908-7916.

Wynn T.A. 2008: Cellular and molecular mechanisms of fibrosis. J. Pathol. 214: 199-210. 